\title{
A NEW PROOF AND A GENERALIZATION OF A THEOREM OF DE BRUIJN
}

\author{
ABDUL AZIZ
}

(Communicated by Irwin Kra)

\begin{abstract}
Using a recently developed interpolation formula, we present elementary new and simple proofs of De Bruijn's theorem and Zygmund's inequality concerning the integral mean estimates for polynomials. We also present a generalization of De Bruijn's theorem which leads to a refinement of a theorem of Erdös and Lax.
\end{abstract}

\section{INTRODUCTION AND STATEMENT OF RESULTS}

Let $P(z)$ be a polynomial of degree $n$ and $P^{\prime}(z)$ its derivative, then

$$
\underset{|z|=1}{\operatorname{Max}}\left|P^{\prime}(z)\right| \leq n \operatorname{Max}_{|z|=1}|P(z)|
$$

and for $q \geq 1$,

$$
\left\{\int_{0}^{2 \pi}\left|P^{\prime}\left(e^{i \theta}\right)\right|^{q} d \theta\right\}^{1 / q} \leq n\left\{\int_{0}^{2 \pi}\left|P\left(e^{i \theta}\right)\right|^{q} d \theta\right\}^{1 / q} .
$$

Inequality (1) is an immediate consequence of S. Bernstein's theorem on the derivative of a trigonometric polynomial (for reference see [6]). Inequality (2) is due to Zygmund [7] who proved it for all trigonometric polynomials of degree $n$ and not only for those which are of the form $P\left(e^{i \theta}\right)$. Inequality (1) can be obtained by letting $q \rightarrow \infty$ in the inequality (2). Both the inequalities (1) and (2) can be sharpened if we restrict ourselves to the class of polynomials having no zeros in $|z|<1$. In this connection P. Erdös conjectured and later P. D. Lax [4] verified that if $P(z) \neq 0$ in $|z|<1$, then (1) can be replaced by

$$
\operatorname{Max}_{|z|=1}\left|P^{\prime}(z)\right| \leq \frac{n}{2} \operatorname{Max}_{|z|=1}|P(z)|,
$$

whereas the corresponding refinement of Zygmund's inequality which was found out by N. G. De Bruijn [2, Theorem 13], is the following:

Received by the editors October 2, 1987 and, in revised form, March 25, 1988.

1980 Mathematics Subject Classification (1985 Revision). Primary 30A10, 30A65; Secondary $30 \mathrm{C} 10$.

Key words and phrases. Derivative of a polynomial, integral mean estimates, inequalities in the complex domain, self-inversive polynomials. 
De Bruijn's Theorem. If $P(z)$ is a polynomial of degree $n$ which has no zeros in the disk $|z|<1$, then for $q \geq 1$,

$$
\left\{\int_{0}^{2 \pi}\left|P^{\prime}\left(e^{i \theta}\right)\right|^{q} d \theta\right\}^{1 / q} \leq n C_{q}\left\{\int_{0}^{2 \pi}\left|P\left(e^{i \theta}\right)\right|^{q} d \theta\right\}^{1 / q}
$$

where

$$
C_{q}=\left\{\frac{1}{2 \pi} \int_{0}^{2 \pi}\left|1+e^{i \alpha}\right|^{q} d \alpha\right\}^{-1 / q} .
$$

The result is best possible and equality in (4) holds for $P(z)=a z^{n}+b$, $|a|=|b|$.

The case $q=2$ was obtained by Lax [4], whereas if we let $q$ tend to infinity in (4), we get Erdös-Lax theorem (inequality (3)). For another proof of De Bruijn's theorem see [5].

In this paper we present apparently new and simple proofs of De Bruijn's theorem and Zygmund's inequality which in essence involve no analysis. The proofs depend on an interpolation formula due to A. Aziz and Q. G. Mohammad [1, Lemma 1] and Minkowski's inequality. We also present a generalization of De Bruijn's theorem. In fact, we use De Bruijn's theorem to prove the following more general result.

Theorem 1. If $P(z)$ is a polynomial of degree $n$ with $\operatorname{Min}_{|z|=1}|P(z)|=m$ and $P(z)$ has no zeros in the disk $|z|<1$, then for every given complex number $\beta$ with $|\beta| \leq 1$ and for $q \geq 1$ we have

$$
\begin{aligned}
& \left\{\int_{0}^{2 \pi} \mid P^{\prime}\left(e^{i \theta}+\left.m n \beta e^{i(n-1) \theta}\right|^{q} d \theta\right\}^{1 / q}\right. \\
& \leq n C_{q}\left\{\int_{0}^{2 \pi}\left|P\left(e^{i \theta}\right)+m \beta e^{i n \theta}\right|^{q} d \theta\right\}^{1 / q}
\end{aligned}
$$

where $C_{q}$ is defined by (5). The result is best possible and equality in (6) holds for $P(z)=a z^{n}+b k^{n},|a|=|b|, k \geq 1$ and $\beta=a /|a|$.

Remark 1. Making $q$ tend to infinity in (6) and choosing argument of $\beta$, with $|\beta|=1$, suitably we obtain that if $P(z) \neq 0$ for $|z|<1$, then

$$
\underset{|z|=1}{\operatorname{Max}}\left|P^{\prime}(z)\right|+n m \leq \frac{n}{2}\left(\operatorname{Max}_{|z|=1}|P(z)|+m\right),
$$

or equivalently

$$
\operatorname{Max}_{|z|=1}\left|P^{\prime}(z)\right| \leq \frac{n}{2}\left(\operatorname{Max}_{|z|=1}|P(z)|-\operatorname{Min}_{|z|=1}|P(z)|\right),
$$

which is an interesting refinement of Erdös-Lax theorem.

\section{LEMMAS}

We require the following interpolation formula [1, Lemma 1]. 
Lemma 1. If $P(z)$ is a polynomial of degree $n$ and $z_{1}, z_{2}, \ldots, z_{n}$ are the zeros of $z^{n}+a$, where $a \neq-1$ is any nonzero complex number, then for any complex number $t$,

$$
t P^{\prime}(t)=\frac{n}{1+a} P(t)+\frac{1+a}{n a} \sum_{k=1}^{n} P\left(t z_{k}\right) \frac{z_{k}}{\left(z_{k}-1\right)^{2}}
$$

and

$$
\sum_{k=1}^{n} \frac{z_{k}}{\left(z_{k}-1\right)^{2}}=-\frac{n^{2} a}{(1+a)^{2}}
$$

With the help of Minkowski's inequality, we deduce from Lemma 1 the following

Lemma 2. If $P(z)$ is a polynomial of degree $n$, then for every real $\alpha$ and $q \geq 1$,

$$
\begin{gathered}
\left\{\int_{0}^{2 \pi}\left|n P\left(e^{i \theta}\right)-e^{i \theta} P^{\prime}\left(e^{i \theta}\right)+e^{i(\alpha+\theta)} P^{\prime}\left(e^{i \theta}\right)\right|^{q} d \theta\right\}^{1 / q} \\
\leq n\left\{\int_{p}^{2 \pi}\left|P\left(e^{i \theta}\right)\right|^{q} d \theta\right\}^{1 / q} .
\end{gathered}
$$

Proof of Lemma 2. By Lemma 1, we have for $|t|=1$,

$$
\begin{aligned}
& \left|n P(t)-t P^{\prime}(t)-a t P^{\prime}(t)\right| \\
& \quad=\left|\frac{(1+a)^{2}}{n a}\right| \mid \sum_{k=1}^{n} P\left(t z_{k}\right) \frac{z_{k}}{\left(z_{k}-1\right)^{2}},
\end{aligned}
$$

where $a \neq-1$ is any nonzero complex number and $z_{k}, k=1,2, \ldots, n$ are the zeros of $z^{n}+a$. We take in particular, $a \neq-1$ to be an arbitrary complex number such that $|a|=1$, then $\left|z_{k}\right|=1$ and $z_{k} \neq 1$ for $k=1,2, \ldots, n$. Moreover it can be easily verified that if $|z|=1, z \neq 1$, then $z /(z-1)^{2}$ is a negative real number and if $|a|=1, a \neq-1$, then $(1+a)^{2} / a$ is a positive real number. Therefore, by (8) it follows that for $|a|=1$ and $a \neq-1$,

$$
\left|\frac{(1+a)^{2}}{n a}\right| \sum_{k=1}^{n}\left|\frac{z_{k}}{\left(z_{k}-1\right)^{2}}\right|
$$

$$
=-\frac{(1+a)^{2}}{n a} \sum_{k=1}^{n} \frac{z_{k}}{\left(z_{k}-1\right)^{2}}=n .
$$


Now from (10) we have by Minkowski's inequality for $q \geq 1$,

$$
\begin{aligned}
& \left\{\int_{0}^{2 \pi}\left|n P\left(e^{i \theta}\right)-e^{i \theta} P^{\prime}\left(e^{i \theta}\right)-a e^{i \theta} P^{\prime}\left(e^{i \theta}\right)\right|^{q} d \theta\right\}^{1 / q} \\
& \quad=\left|\frac{(1+a)^{2}}{n a}\right|\left\{\int_{0}^{2 \pi}\left|\sum_{k=1}^{n} P\left(e^{i \theta} z_{k}\right) \frac{z_{k}}{\left(z_{k}-1\right)^{2}}\right|^{q} d \theta\right\}^{1 / q} \\
& \leq\left|\frac{(1+a)^{2}}{n a}\right| \sum_{k=1}^{n}\left|\frac{z_{k}}{\left(z_{k}-1\right)^{2}}\right|\left\{\int_{0}^{2 \pi}\left|P\left(e^{i \theta} z_{k}\right)\right|^{q} d \theta\right\}^{1 / q} \\
& =\left|\frac{(1+a)^{2}}{n a}\right| \sum_{k=1}^{n}\left|\frac{z_{k}}{\left(z_{k}-1\right)^{2}}\right|\left\{\int_{0}^{2 \pi}\left|P\left(e^{i \theta}\right)\right|^{q} d \theta\right\}^{1 / q} .
\end{aligned}
$$

Using (11) and writing $a=-e^{i \alpha}$ where $\alpha \not \equiv 0(\bmod 2 \pi)$, we obtain

$$
\begin{aligned}
& \left\{\int_{0}^{2 \pi}\left|n P\left(e^{i \theta}\right)-e^{i \theta} P^{\prime}\left(e^{i \theta}\right)+e^{i(\alpha+\theta)} P^{\prime}\left(e^{i \theta}\right)\right|^{q} d \theta\right\}^{1 / q} \\
& \leq n\left\{\int_{0}^{2 \pi}\left|P\left(e^{i \theta}\right)\right|^{q} d \theta\right\}^{1 / q} .
\end{aligned}
$$

Since this inequality is obviously true for $\alpha \equiv 0(\bmod 2 \pi)$, therefore, Lemma 2 is proved.

\section{Proofs of the theorems}

Proof of De Bruijn's theorem. Since the polynomial $P(z)$ does not vanish in the disk $|z|<1$, it follows by a simple argument (see [1, inequality (9)]) that

$$
\left|P^{\prime}(z)\right| \leq\left|n P(z)-z P^{\prime}(z)\right| \text { for }|z|=1 .
$$

By Lemma 2, we have for every real $\alpha$ and $q \geq 1$,

$$
\int_{0}^{2 \pi}\left|F(\theta)+e^{i \alpha} G(\theta)\right|^{q} d \theta \leq n^{q} \int_{0}^{2 \pi}\left|P\left(e^{i \theta}\right)\right|^{q} d \theta
$$

where

$$
F(\theta)=n P\left(e^{i \theta}\right)-e^{i \theta} P^{\prime}\left(e^{i \theta}\right) \text { and } \quad G(\theta)=e^{i \theta} P^{\prime}\left(e^{i \theta}\right) .
$$

Integrating both sides of (13) with respect to $\alpha$ from 0 to $2 \pi$, we get for $q \geq 1$,

$$
\int_{0}^{2 \pi} \int_{0}^{2 \pi}\left|F(\theta)+e^{i \alpha} G(\theta)\right|^{q} d \alpha d \theta \leq 2 \pi n^{q} \int_{0}^{2 \pi}\left|P\left(e^{i \theta}\right)\right|^{q} d \theta .
$$

Now for every real $\alpha$ and $r \geq 1$, we have $\left|r+e^{i \alpha}\right| \geq\left|1+e^{i \alpha}\right|$, which implies

$$
\int_{0}^{2 \pi}\left|r+e^{i \alpha}\right|^{q} d \alpha \geq \int_{0}^{2 \pi}\left|1+e^{i \alpha}\right|^{q} d \alpha, \quad q>0 .
$$


If $G(\theta) \neq 0$, we take $r=|F(\theta)| /|G(\theta)|$, then by (12), $r \geq 1$ and we get

$$
\begin{aligned}
\int_{0}^{2 \pi}\left|F(\theta)+e^{i \alpha} G(\theta)\right|^{q} d \alpha & =|G(\theta)|^{q} \int_{0}^{2 \pi}\left|\frac{F(\theta)}{G(\theta)}+e^{i \alpha}\right|^{q} d \alpha \\
& =|G(\theta)|^{q} \int_{0}^{2 \pi}|| \frac{F(\theta)}{G(\theta)}\left|+e^{i \alpha}\right|^{q} d \alpha \\
& \geq\left|P^{\prime}\left(e^{i \theta}\right)\right|^{q} \int_{0}^{2 \pi}\left|1+e^{i \alpha}\right|^{q} d \alpha .
\end{aligned}
$$

If $G(\theta)=0$, then $P^{\prime}\left(e^{i \theta}\right)=0$ and this inequality is trivially true. Using this in (14), we conclude that for $q \geq 1$,

$$
\int_{0}^{2 \pi}\left|1+e^{i \alpha}\right|^{q} d \alpha \int_{0}^{2 \pi}\left|P^{\prime}\left(e^{i \theta}\right)\right|^{q} d \theta \leq 2 \pi n^{q} \int_{0}^{2 \pi}\left|P\left(e^{i \theta}\right)\right|^{q} d \theta,
$$

which immediately leads to (4) and this completes the proof of De Bruijn's theorem.

Proof of Theorem 1. By hypothesis, the polynomial $P(z)$ does not vanish in $|z|<1$ and $m=\operatorname{Min}_{|z|=1}|P(z)|$, therefore, $m \leq|P(z)|$ for $|z| \leq 1$. We first show that for any given complex number $\beta$ with $|\beta| \leq 1$, the polynomial $F(z)=P(z)+m \beta z^{n}$ does not vanish in $|z|<1$. This is obvious if $m=0$ and for $m>0$ we prove it by contradiction. Assume that $F(z)$ has a zero in $|z|<1$, say at $z=z_{0}$ with $\left|z_{0}\right|<1$, then we have

$$
P\left(z_{0}\right)+m \beta z_{0}^{n}=F\left(z_{0}\right)=0 .
$$

This gives

$$
\left|P\left(z_{0}\right)\right|=\left|m \beta z_{0}^{n}\right| \leq m\left|z_{0}\right|^{n}<m,
$$

which is clearly a contradiction (to the minimum modulus principle). Hence $F(z)$ has no zeros in the disk $|z|<1$ for every $\beta$ with $|\beta| \leq 1$. Applying now De Bruijn's theorem to the polynomial $F(z)=P(z)+m \beta z^{n}$, we readily obtain (6) and this proves Theorem 1.

Proof of Zygmund's inequality. We take $a=1$ in Lemma 1 to get for $|t|=1$,

$$
\left|P^{\prime}(t)\right|=\left|\frac{n}{2}(t)+\frac{2}{n} \sum_{k=1}^{n} P\left(t z_{k}\right) \frac{z_{k}}{\left(z_{k}-1\right)^{2}}\right|,
$$

where $z_{k}, k=1,2, \ldots, n$ are the zeros of $z^{n}+1$. Hence by using Minkowski's inequality, we obtain for $q \geq 1$,

$$
\begin{aligned}
& \left\{\int_{0}^{2 \pi}\left|P^{\prime}\left(e^{i \theta}\right)\right|^{q} d \theta\right\}^{1 / q} \leq \frac{n}{2}\left\{\int_{0}^{2 \pi}\left|P\left(e^{i \theta}\right)\right|^{q} d \theta\right\}^{1 / q} \\
& +\frac{2}{n} \sum_{k=1}^{n}\left|\frac{z_{k}}{\left(z_{k}-1\right)^{2}}\right|\left\{\int_{0}^{2 \pi}\left|P\left(e^{i \theta} z_{k}\right)\right|^{q} d \theta\right\}^{1 / q} \\
& \quad=\left\{\frac{n}{2}+\frac{2}{n} \sum_{k=1}^{n}\left|\frac{z_{k}}{\left(z_{k}-1\right)^{2}}\right|\right\}\left\{\int_{0}^{2 \pi}\left|P\left(e^{i \theta}\right)\right|^{q} d \theta\right\}^{1 / q} .
\end{aligned}
$$


Since $\left|z_{k}\right|=1, z_{k} \neq 1, k=1,2, \ldots, n$ and therefore, with the help of (8) (for $a=1$ ) we get

$$
\sum_{k=1}^{n}\left|\frac{z_{k}}{\left(z_{k}-1\right)^{2}}\right|=-\sum_{k=1}^{n} \frac{z_{k}}{\left(z_{k}-1\right)^{2}}=\frac{n^{2}}{4} .
$$

Using this in (15), we obtain for $q \geq 1$,

$$
\left\{\int_{0}^{2 \pi}\left|P^{\prime}\left(e^{i \theta}\right)\right|^{q} d \theta\right\}^{1 / q} \leq n\left\{\int_{0}^{2 \pi}\left|P\left(e^{i \theta}\right)\right|^{q} d \theta\right\}^{1 / q},
$$

which is (2) and this completes the proof of Zygmund's inequality.

Remark 2. If $P(z)$ is a self-inversive polynomial, i.e. if $P(z)=z^{n} \overline{P(1 / \bar{z})}$ then it can be easily seen that $\left|P^{\prime}(z)\right|=\left|n P(z)-z P^{\prime}(z)\right|$ for $|z|=1$, so that

$$
|F(\theta) / G(\theta)|=\left|\left(n P\left(e^{i \theta}\right)-e^{i \theta} P^{\prime}\left(e^{i \theta}\right)\right) / e^{i \theta} P^{\prime}\left(e^{i \theta}\right)\right|=1 .
$$

Using this in (14) and proceeding similarly as in the proof of De Bruijn's theorem, we conclude that if $P(z)$ is a self-inversive polynomial of degree $n$, then for every $q \geq 1$ we have

$$
\left\{\int_{0}^{2 \pi}\left|P^{\prime}\left(e^{i \theta}\right)\right|^{q} d \theta\right\}^{1 / q} \leq n C_{q}\left\{\int_{0}^{2 \pi}\left|P\left(e^{i \theta}\right)\right|^{q} d \theta\right\}^{1 / q},
$$

where $C_{q}$ is defined by (5). The result is best possible and equality in (16) holds for $P(z)=z^{n}+1$. This result was earlier noted by Dewan and Govil [3] also.

\section{REFERENCES}

1. A. Aziz and Q. G. Mohammad, Simple proof of a theorem of Erdös and Lax, Proc. Amer. Math. Soc. 80 (1980), 119-122.

2. N. G. De Bruijn, Inequalities concerning polynomials in the complex domain, Nederl. Akad. Wetensch. Proc. 50 (1947), 1265-1272 = Indag. Math. 9 (1947), 591-598.

3. K. K. Dewan and N. K. Govil, An inequality for self-inversive polynomials, J. Math. Anal. Appl. 95 (1983), 490.

4. P. D. Lax, Proof of a conjecture of P. Erdös on the derivative of a polynomial, Bull. Amer. Math. Soc. 50 (1944), 509-513.

5. Q. I. Rahman, Functions of exponential type, Trans. Amer. Math. Soc. 135 (1969), 295-309.

6. A. C. Schaeffer, Inequalities of A. Markoff and S. Bernstein for polynomials and related functions, Bull. Amer. Math. Soc. 47 (1941), 565-579.

7. A. Zygmund, A remark on conjugate series, Proc. London Math. Soc. (2) 34 (1932), 392-400.

Department of Mathematics, University of Kashmir, Hazratbal SRinagar-190006, KASHMIR, INDIA 